\title{
Improving ultrasonic imaging of the vascular wall and blood flow using a multiphysics simulation tool integrating fluid-structure interaction and ultrasound simulations
}

\author{
Abigail Swillens \\ Patrick Segers \\ IBiTech-bioMMeda \\ Ghent University, Ghent, Belgium \\ Email: abigail.swillens@ugent.be \\ Joris Degroote \\ Jan Vierendeels \\ Department of flow, heat \\ and combustion mechanics \\ Ghent University, Ghent, Belgium
}

\author{
Lasse Lovstakken \\ Department of Circulation and Medical Imaging \\ NTNU, Trondheim, Norway \\ St. Olavs University Hospital, Trondheim, Norway
}

\begin{abstract}
The currently applied ultrasonic blood flow and vessel wall imaging methods still demonstrate several limitations. To support the development of new vascular ultrasonic imaging modalities, we developed a simulation environment integrating ultrasound (US) and fluid-structure interaction (FSI) simulations, allowing construction of synthetic US-images based on physiologically realistic behavior of an artery. An in-house FSI-code was developed to strongly couple the flow solver Fluent and structural solver Abaqus; US-simulations were performed with Field II. A distensible tube, representing the common carotid artery was simulated. FSI and US-simulations were coupled by seeding scatterers in the fluid and structural domain and propagating them during the simulated scan procedure based on flow and structural displacement fields from FSI. Simulations yielded raw RF-data, which were further processed for arterial wall distension and shear rate imaging. Our simulations demonstrated that (i) the wall distension application is sensitive to measurement location (highest distension found when tracking the intimalumen transition); (ii) strong reflections between tissue transitions can potentially cloud a correct measurement; (iii) maximum shear rate was underestimated during the complete cardiac cycle, with largest discrepancy during peak systole; (iv) due to difficulties measuring near-wall velocities with US, shear rate reached its maximal value at a distance from the wall. We conclude that our FSI-US simulation environment provides realistic RF-signals which can be processed into ultrasoundderived medical images and measurements.
\end{abstract}

\section{INTRODUCTION}

Ultrasonic echography is still the preferred method for clinical screening of atherosclerosis in large, superficial arteries. In particular, the carotid artery is often investigated in clinical protocols since the artery directly supplies blood to the brain and the location is prone to development of atherosclerosis. However, most commonly used clinical blood flow imaging modalities are still limited to 1D measurements since only the velocity component in the direction of the ultrasound (US) beam is visualized. Other imaging modalities aim to assess arterial stiffness, but here the current methods are also hampered. A common application is measuring tissue velocities using Doppler based methods, which is limited to
1D visualization as well. Often, tissue velocities are further integrated to assess vessel distension. However, this provides information regarding vessel kinematics rather than vessel mechanics.

We recently developed a computer simulation tool [1] which integrates computational fluid dynamics (CFD) with US simulation software (Field II; [2], [3]), providing a flexible tool for the validation and development of US blood flow modalities in the presence of complex flow. The US echoes are simulated by modeling blood as a random point scatterer distribution on which the US waves reflect. The scatterer positions are updated using the CFD-velocity fields interpolated in space and time.

An important limitation of our work was the absence of the moving vessel wall in our model, which influences flow visualization through the motion of the boundaries of the fluid domain, as well as via US echoes generated by the vessel wall. Fluid-structure interaction (FSI) simulations allow for the coupled computation of blood flow and arterial wall mechanics [4]. It is therefore a natural extension of our work to also seed scatterers in the vessel wall, and to use FSI-simulations to calculate scatterer positions in both the blood flow and vessel wall.

The aim of this paper is two-fold. We first present a method providing scatterer phantoms of both the blood flow and arterial wall, i.e. a virtual echographic phantom of a straight arterial segment. Subsequently, we illustrate the potential of the model. As a first application, the US measurement of vessel distension will be investigated [5]. We will use the RFdata resulting from scatterers in the arterial wall to assess the performance of a previously used vessel wall-tracking algorithm. In a second application, we will study to what extent it is possible to measure shear rate profiles using Doppler-based measurements along one scanline. As earlier described by Brands et al. [6], shear rate profiles can be derived from US flow velocity profiles. However, due to difficulties in measuring low velocities in the vicinity of the moving wall, the maximal shear rate is measured at a certain 
distance from the wall, while the actual maximal shear rate might be differing from this position and in magnitude. To the best of our knowledge, the relationship between ground-truth and US-derived shear rate has never been studied in detail.

\section{Methods}

\section{A. An ultrasound simulation environment based on FSI}

The RF-signals from the vessel wall and blood were simulated using the Field II software created by Jensen et al. This simulation software allows modeling arbitrary US transducers and realistic image scan sequencing. Using linear system theory, the ultrasound field is determined based on the ultrasonic excitation pulse, the temporal impulse responses of the transmitting and receiving transducers, and the spatial impulse response at a given point. Field II models tissue as a collection of random point scatterers. The required scatterer density is related to the imaging system resolution, with 10 scatterers per resolution cell assuring Gaussian distributed RFsignals. The scattering strength is modeled using a normal distribution of scattering amplitudes with mean and standard deviation varying according to the tissue properties. Dynamic objects (i.e. blood flow and moving vessel wall) are achieved by moving the point scatterers during simulation. Each US beam is simulated individually, and it is therefore possible to update the position of moving scatterers between beam acquisitions.

We previously developed a method to generate scatterers for Field II simulations based on CFD-calculations with rigid walls [1]. For distensible fluid geometries (as in FSIsimulations), straightforward linear temporal interpolation of the velocity fields is however not possible. We therefore followed an approach where the scatterer displacement is approximated by updating scatterer velocities for each FSItimestep. To avoid that scatterers are displaced outside the fluid domain in a shrinking geometry or that voids are created in an expanding geometry, scatterers are displaced using the velocity vector from the future FSI time step, with the velocity vector extracted from an approximated mapped position at that time step. This approach is justified due to the backward Euler time discretization used by the flow solver. It provides correct displacements for scatterers at the fluid-structure interface, but it is an approximated approach within the flow field.

The structure phantom generation is less complex because of the Lagrangian grid formulation, i.e. the grid displacement corresponds with the material displacement and hence also with the scatterer displacement. However, the vessel wall needs more refined scatterer generation due to its complex composition, with flexibility of defining different scattering properties in different vessel regions. Therefore, the mesh was divided into 3 layers of hexahedrons, with scatterer properties easily modifiable for each layer. This represents to some extent the intima, media and adventitia of an artery, although we assumed equal thickness of each layer, while this is not the case in vivo. Besides these random scatterers, we also mimicked the specular reflections at the transition regions between different tissue types (i.e. tissue/vessel wall and vessel wall/blood) by placing scatterers at fixed distances along these interfaces. These mimicked specular reflections had a higher intensity than the scattering from the random tissue and blood scatterers. Details on the scatterer properties can be found in table 1 .

\section{B. Fluid-structure interaction simulations}

A straight elastic tube with inner radius $3 \mathrm{~mm}$, outer radius 4 $\mathrm{mm}$, and length $5 \mathrm{~cm}$ was simulated. The vessel wall was modeled using a linearized elastic material model with a Young modulus of $250 \mathrm{kPa}$, Poisson modulus of 0.49 , and density $1200 \mathrm{~kg} / \mathrm{m}^{3}$. Linearization was performed with respect to a reference pressure and inner radius of respectively $10 \mathrm{mmHg}$ and $3 \mathrm{~mm}$. Circumferential and longitudinal movement of the tube was prevented. Blood was modeled as a Newtonian liquid with a viscosity of $3.5 \mathrm{mPas}$ and a density of $1050 \mathrm{~kg} / \mathrm{m}^{3}$. A velocity profile was measured in the common carotid artery of a healthy volunteer using ultrasonic pulsed wave Doppler $(12 \mathrm{~L}$ linear array vascular probe, GE Medical Systems, Milwaukee, WI, USA) and was applied as a mass flow inlet condition. The outlet boundary condition was a non-invasively measured pressure waveform, scaled with a pulse pressure of $40 \mathrm{mmHg}$, representative of a healthy hemodynamical condition. The fluid mesh consisted of 34400 triangular prisms and was adapted to the position of the fluid-structure interface with a spring analogy. The structural domain was composed of 720 quadratic continuum hexahedrons (20 nodes), which allowed layered modeling of the vessel wall. The cardiac cycle of 1s was divided into timesteps of $5 \mathrm{~ms}$ and 2 cycles were computed to obtain results independent of transient effects.

FSI-simulations were performed in a partitioned way, computing the flow and structural equations with a separate flow and structural solver. An in-house code 'Tango' was used to couple the flow solver Fluent (Ansys, Canonsburg, PA, USA) and the structural solver Abaqus (Simulia, Inc., Providence, RI, USA). In particular, Dirichlet-Neumann partitioning was used (flow problem is solved for a given displacement of the fluid-structure interface; structural problem is solved for a stress boundary condition applied on wet side of the structure). To enhance convergence of the coupling iterations, an Interface Quasi-Newton method was used, which replaces the complex fluid or solid solver on the interface by approaching the Jacobian of the solver on the interface. Further, an Arbitrary Lagrangian Eulerian (ALE) method was used to match the different grid formulations for the fluid and structural domain.

TABLE I

ASSIGNED SCATTERER PROPERTIES.

\begin{tabular}{|c|c|c|}
\hline & Mean amplitude & STD \\
\hline Inner wall (specular reflections) & 0 & 1 \\
\hline Outer wall (specular reflections) & 0 & 0.01 \\
\hline Wall & 50 & 0.01 \\
\hline Blood & 100 & 0.01 \\
\hline
\end{tabular}


TABLE II

SIMULATION SETUP FOR THE BLOOD FLOW AND VESSEL WALL IMAGING APPLICATION

\begin{tabular}{|c|c|c|}
\hline & Blood flow & Wall distension \\
\hline$f_{0}$ & $5 \mathrm{MHz}$ & $8 \mathrm{MHz}$ \\
\hline Element Pitch & $245 \mu \mathrm{m}$ & $200 \mu \mathrm{m}$ \\
\hline Element Height & $6 \mathrm{~mm}$ & $3.3 \mathrm{~mm}$ \\
\hline Focus & $2 \mathrm{~cm}$ & $2 \mathrm{~cm}$ \\
\hline Dynamic receive focusing & yes & yes \\
\hline Expanding aperture & yes & yes \\
\hline Excitation & sinusoidal & sinusoidal \\
\hline Pulseperiods & 4 & 1.5 \\
\hline$P R F_{\max }$ & $8000 \mathrm{~Hz}$ & $8000 \mathrm{~Hz}$ \\
\hline PRF & $8000 \mathrm{~Hz}$ & $1000 \mathrm{~Hz}$ \\
\hline Packetsize & 64 & 3 \\
\hline
\end{tabular}

\section{Assessing arterial distensibility}

In a first application, we simulate the US measurement of vessel distension, where the US beam is transmitted perpendicular to the vessel wall. Vessel wall motion is tracked by integrating wall velocities: $z[t+\Delta t]=z[t]+v \Delta t$ with $\mathrm{z}[\mathrm{t}]$ the position in the vessel wall, $\mathrm{v}[\mathrm{t}]$ the axial velocity as estimated by US (modified autocorrelation approach), and $\Delta \mathrm{t}$ the temporal resolution corresponding to the packet size times the pulse repetition period $(3 \cdot 1 / 1000)$. Only the RFdata of the arterial wall are processed for this application, hence neglecting a potential effect of the flow motion on the wall velocity estimator. A 12L linear array probe (GE Medical Systems, Milwaukee, WI, USA), as used in the applied distension software [5], was modeled with a 1.5 period sinusoidal pulse excitation of $8 \mathrm{MHz}$ centre frequency. Due to the lower velocity range of tissue compared to blood, a lower pulse repetition frequency (PRF) of $1 \mathrm{kHz}$ was applied.

\section{Wall shear rate imaging}

For the flow (shear rate) application, the tube phantom was angled 70 degrees with respect to the US beam (=axial) direction. Velocity profiles in the axial direction were obtained using an autocorrelation algorithm. Note that only the RFsignal from the blood was used to estimate flow velocities. A 4-period sinusoidal excitation pulse with $5 \mathrm{MHz}$ centre frequency was chosen. Velocity profiles halfway the tube were obtained using a packet of 64 pulses emitted with a PRF of 8 $\mathrm{kHz}$, resulting in 120 frames for the complete cardiac cycle. A sliding window averaging filter was used. In the results, displayed velocities are angle-corrected velocities and thus represent velocities along the axis of the tube. A complete overview of the simulation parameters can be found in table 2 .

\section{RESULTS}

\section{A. Ultrasonic assessment of arterial distensibility}

RF ultrasound data for the wall were generated from the FSI-US coupling procedure, and are displayed as an M-Mode
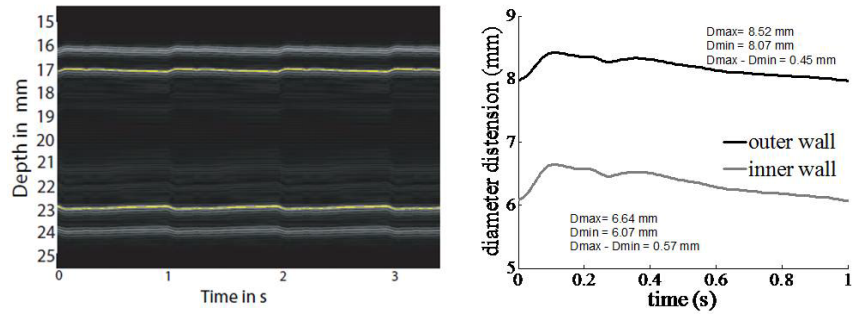

Fig. 1. Left: M-mode display of RF-data designating arterial wall motion. Right: diameter distension waveforms after tracking the motion of the lumenintima (inner wall) and media-adventitia (outer wall) transition.

image in Fig.1. Using the distension software of Rabben et al. [5], the motion of the anterior and posterior intima-lumen (inner wall tracking, see Fig 1.) and media-adventitia (outer wall tracking) was tracked. Subtracting these tracking curves yielded the vessel diameter distension waveforms, as displayed in Fig.1. Using inner wall tracking data, arterial distension $\Delta \mathrm{D} / \mathrm{D}(\Delta \mathrm{D}=\mathrm{Dmax}-\mathrm{Dmin}$ and $\mathrm{D}=\mathrm{Dmin})$ was $9 \%$, which is representative for the distension of the common carotid artery of a healthy adult.

In fig. 2, we further analyzed arterial distension using the circumferential strain $\Delta \mathrm{D} / \mathrm{D}$, which is frequently used in clinical practice as a measure of arterial distensibility. Using the simulated RF-data, the circumferential strain $\Delta \mathrm{D} / \mathrm{D}$ was computed at several depths within the wall (dashed line in fig. 2). Assuming an incompressible material and neglecting deformation along the vessel axis, the theoretical $\Delta \mathrm{D} / \mathrm{D}$ - curve was also computed and indicated by the solid line. While the circumferential strain $\Delta \mathrm{D} / \mathrm{D}$ obtained from theoretical calculation as well as FSI computations resulted in a $1 / D^{2}$ -relationship, this was not the case for the wall tracking based on the FSI-US coupling procedure. Interestingly, there was a very good match between theory and ultrasound wall tracking based on the FSI-US data of the inner and outer

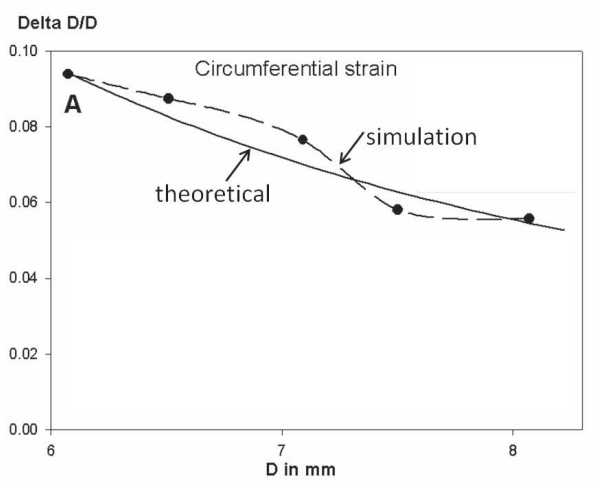

Fig. 2. The circumferential strain $\Delta \mathrm{D} / \mathrm{D}$ obtained with the FSI-US coupling procedure (dashed line) and compared to the theoretical relationship (solid line). 

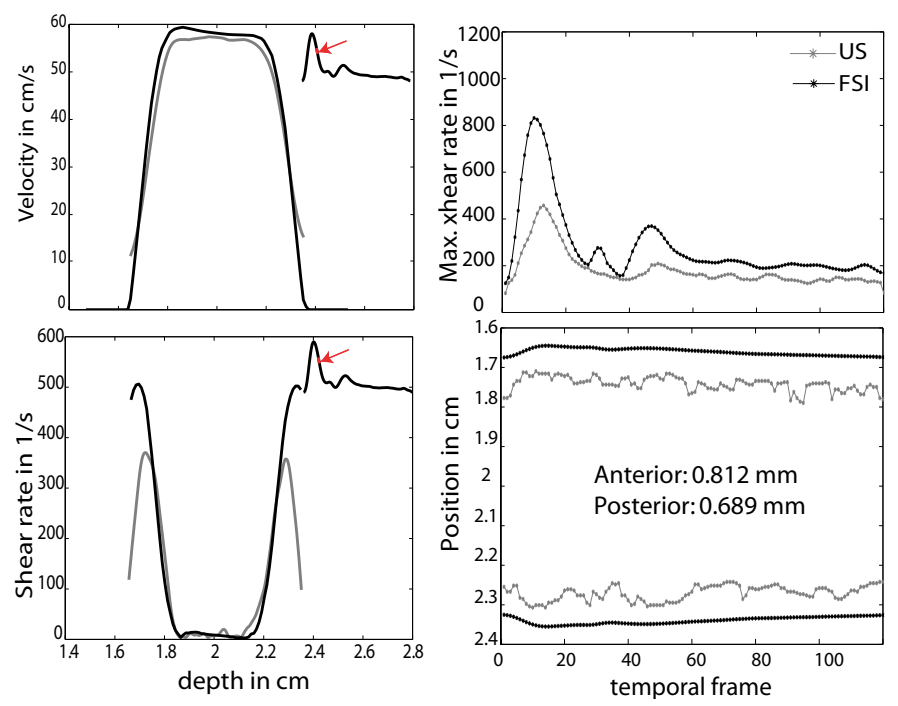

Fig. 3. Left: angle-corrected velocity and shear rate profile for FSI (black) and US (gray) data, during systolic deceleration. Right: comparison between peak shear rate directly derived from the FSI-data and obtained from the ultrasound data (top) and display of the position where maximum shear rate is found with FSI and US.

wall tracking. However, tracking based on points within the vessel wall resulted in an $\mathrm{S}$-shaped relationship between $\Delta \mathrm{D} / \mathrm{D}$ and depth in the arterial wall. This curve flattening can be explained by the strong reflections present near the vessel wall boundaries, which blur the velocity measurement in its immediate neighborhood. Hence, deducing deformation trends and material properties of the studied vessel wall tissue based solely on wall tracking measurements within the arterial wall may lead to flawed interpretations.

\section{B. Ultrasonic assessment of wall shear rate}

Fig.3 (left panels) shows the velocity and shear rate profile during systolic deceleration, as obtained from the FSI-US coupling procedure and compared to the FSI ground truth. The upper right panel demonstrates how the measured (from multiphysical simulations) and true maximal shear rate vary during the cardiac cycle. From fig.3, it is obvious that FSI-US shear rate reaches its maximum value at a certain distance from the wall. FSI-US derived maximal shear rate is systematically lower than the reference value derived from the FSI simulations, with the largest discrepancy appearing near peak systole. There was no clear relation between the location of maximal shear rate and the actual position of the arterial wall (lower right panel fig.3). However, one can notice that the position of the US-derived maximal shear rate shows an asymmetrical trend compared to the vessel centre: maximal shear rate is measured closer to the posterior wall than to the anterior wall.

\section{Discussion}

Although the multiphysics model as presented in this study represents a major improvement over our existing model, limitations are still present, both at the biomechanical and acoustical level. More realistic material behavior including anisotropic and hyperelastic material properties could have been modeled (at the expense of higher computational times). The influence of the surrounding tissue on the vessel wall movement, residual stresses and effects of longitudinal prestretch of the structure could be taken into account in future work. Further, the boundary conditions to solve the fluid problem could be refined by applying a physiologically realistic vascular impedance as outlet boundary condition, inducing more realistic wave propagation phenomena in the tube. The Field II method does not account for nonlinear wave propagation of ultrasound waves or multiple scattering. We did not include noise or frequency dependent scattering in our simulations. Furthermore, the tissue echogenic properties are simulated as a combination of random scatterers in the wall and mimicked specular reflections along the inner and outer boundary of the wall. Although this results in realistically looking RF spectra, further fine-tuning and optimization towards RF-spectra of actual tissue may be mandatory. It should also be stressed that the distension data are solely based on RFsignals from the wall, while the shear rate application relied only on RF-signals from the flow. The shown applications should therefore mainly be considered as a demonstration of the potential of the simulation tool in developing vascular imaging tools, rather than a thorough validation of each of these applications. It is, for that matter, possible that the imaging and signal processing setups can be further optimized for each specific application. We conclude that our method to couple fluid-structure interaction and ultrasound simulations provides realistic radio-frequent signals from both the tissue and the blood pool which can be processed into ultrasoundderived medical images and measurements. Further research will focus on applications for the ultrasonic investigation of the carotid bifurcation.

\section{REFERENCES}

[1] A. Swillens, L. Lovstakken, J. Kips, H. Torp, and P. Segers, "Ultrasound simulation of complex flow velocity fields based on computational fluid dynamics," IEEE Transactions on Ultrasonics, Ferroelectrics and Frequency Control, vol. 56, no. 3, pp. 546-556, 2009.

[2] J. A. Jensen and N. B. Svendsen, "Calculation of pressure fields from arbitrarily shaped, apodized, and excited ultrasound transducers," IEEE transactions on Ultrasonics, Ferroelectrics, and Frequency control, vol. 39, no. 2, pp. 262-267, 1992.

[3] J. A. Jensen, "Field: A program for simulating ultrasound systems," Medical and Biological Engineering and Computing, vol. 34, pp. 351352, 1996.

[4] J. Degroote, K. J. Bathe, and J.Vierendeels, "Performance of a new partitioned procedure versus a monolithic procedure in fluid-structure interaction," Computers \& Structures, vol. 87, p. 793801, 2009.

[5] S. I. Rabben, S. Bjaerum, V. Sorhus, and H. Torp, "Ultrasound based vessel wall tracking: an autocorrelation technique with rf center frequency estimation," Ultrasound in Med. \& Biol., vol. 28, pp. 507-517, 2002.

[6] P. J. Brands, A. P. G. Hoeks, L. Hofstra, and R. S. Reneman, "A noninvasive method to estimate wall shear rate using ultrasound," Ultrasound in Med. \& Biol., vol. 21, pp. 171-185, 1994. 